\title{
A Hawaiian mountain climate cross-section
}

\author{
Dennis Nullet, James O. Juvik, Alys Wall \\ Department of Geography, University of Hawai'i at Hilo, Hilo, Hawai'i 96720, USA
}

\begin{abstract}
This study analyzes 2 yr of surface climatological measurements at 6 sites forming a windward/leeward mountain cross-section on Hawai'i Island. Sites range in elevation from sea level to $2600 \mathrm{~m}$ and include wet, windward and dry, leeward slopes below the trade wind temperature inversion and a high elevation site located in the cool, dry air above the inversion. Climate contrasts between sites are emphasized using monthly average values. Data for the $670 \mathrm{~m}$ elevation leeward site represent some of the first climatological measurements of a unique leeward transition zone between areas of summer and winter rainfall maxima.
\end{abstract}

KEY WORDS: Hawaii $\cdot$ Mountain · Climate

\section{INTRODUCTION}

Only recently have climatologists begun detailed climate studies of Hawaiian monntains While the general circulation and rainfall patterns have long been known, few meteorological stations had been emplaced and maintained in mountainous areas for any length of time, with the exception of Mauna Loa Observatory (MLO) at $3400 \mathrm{~m}$ on Hawai'i Island. Since 1980, however, the results of a number of climatological networks have been reported covering a wide variety of sensors. Ekern (1983) reported several years of data, including evaporation and solar radiation, for transects into the Ko'olau mountains on 'Ōahu. Giambelluca \& Nullet (1991) maintained a transect between 950 and $3000 \mathrm{~m}$ on leeward Haleakalā, Maui, for several years, primarily to study the climate through the trade wind inversion. Nullet (1989) summarized several years of measurements between sea level and $1650 \mathrm{~m}$ that had been gathered for agricultural research on the northwest flank of Haleakalā. Chen \& Nash (1994) and Chen \& Wang (1994) analyzed $40 \mathrm{~d}$ of measurements at 50 sites on the island of Hawai'i giving a comprehensive picture of the island's surface airflow, rainfall, temperature, and humidity patterns under summer trade wind conditions. Juvik et al. (1993) and Juvik \& Nullet (1994) reported the results of a climate transect through the wet windward rainforest and the climate of a high elevation dry forest on the island of Hawai'i. The current study expands the data presented in the latter 2 articles and includes a new site at $670 \mathrm{~m}$ on leeward Hawai'i, a site representing the area between Mauna Loa and Hualālai (which) has little wcather information (Schroeder 1993)', lying in a unique transition zone between areas of summer and winter rainfall maxima.

While the data presented here characterize the climate of mountains in Hawai'i, the results should have direct analogies on other high volcanic islands in the trade wind belts with little measured data, such as Tenerife $(3718 \mathrm{~m})$ in the Canary Islands, Fogo (2829 m) in the Cape Verde Islands, and La Reunion (3069 m) in the Mascarene Islands.

\section{STUDY SITE AND MEASUREMENTS}

The 6 meteorological stations are located between sea level and $2600 \mathrm{~m}$ on the island of Hawai'i (Fig. 1), which lies at the southeast end of the Hawaiian Island chain at approximately $19^{\circ}$ to $20^{\circ} \mathrm{N}, 155^{\circ}$ to $156^{\circ} \mathrm{W}$. Over the open ocean, northeast trade winds prevail 80 to $95 \%$ of the time in summer (May through September) but diminish in frequency during winter to as little as $50 \%$ in January (Blumenstock \& Price 1967). The trade wind inversion (a subsidence temperature inversion in the descending limb of the Hadley cell circulation present $70 \%$ of the time at 1800 to $2400 \mathrm{~m}$ ) suppresses cloud development and limits average annual open ocean rainfall to $500-600 \mathrm{~mm}$. Three large shield 


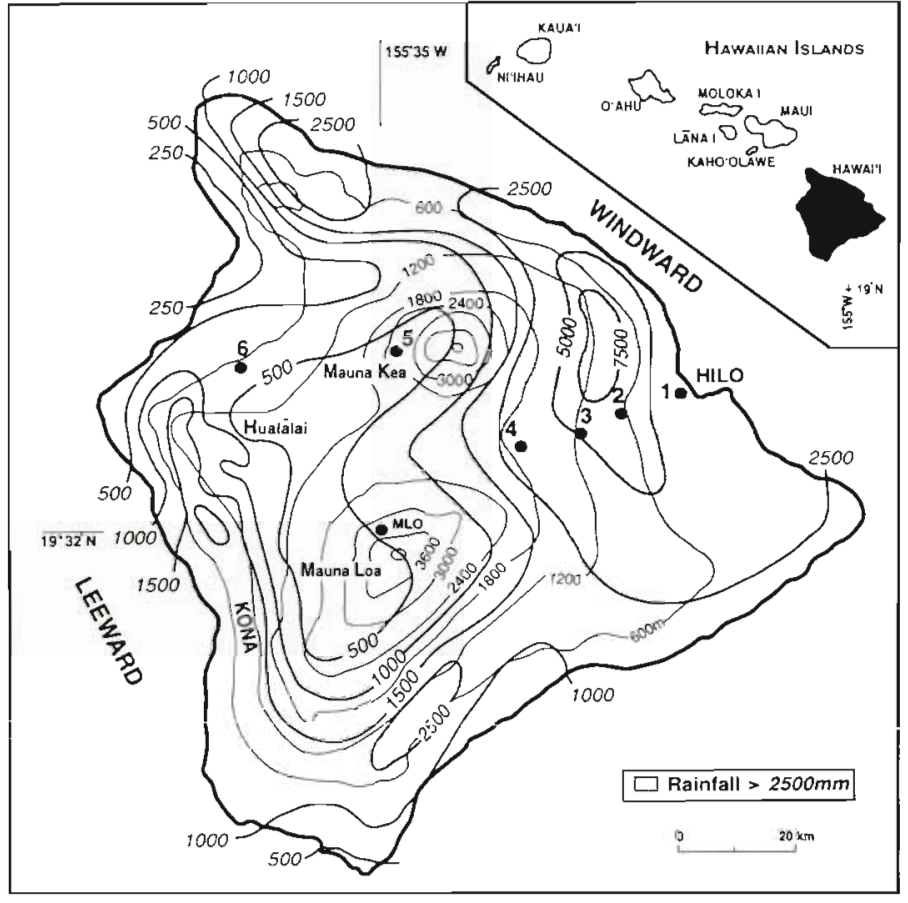

Fig. 1. Station locations, Hawai'i. Rainfall (mm; thick contour lines) from Giambelluca et al. (1986) and Armstrong (1983). 1: UH-Hilo $(55 \mathrm{~m})_{i}$ 2: Lower Saddle $(710 \mathrm{~m})_{i}$ 3: Middle Saddle $(1130 \mathrm{~m})_{i} 4$ : Upper Saddle (1650 m); 5: Pu'u La'u (2600 m); 6: Pu'uanahulu $(670 \mathrm{~m})$. MLO: Mauna Loa Observatory (3400 m). Elevations in $\mathrm{m}$ (thin contour lines)

volcanoes on Hawai'i rise well above the usual level of the inversion and thus host distinctly separate climates above (cool and dry) and below (warm and humid) that layer. In addition, the interaction of the mountains and prevailing trade wind flow generates wet windward slopes and a dry leeward rainshadow. The cross-section of stations included in this report includes measurements in the major climate zones: a wet, windward transect from sea level to the inversion level and dry, leeward sites above and below the inversion (see Fig. 1).

Each automated meteorological station (Campbell Scientific, Logan, UT, USA) recorded global solar radiation, photosynthetically active radiation, air temperature and relative humidity at $2 \mathrm{~m}$, wind speed and direction at $3 \mathrm{~m}$, soil temperature at $1 \mathrm{~cm}$, leaf wetness, and rainfall. Net radiation was recorded at all but the Upper and Middle Saddle sites. Hourly maxima and minima were recorded as well as hourly totals for rainfall and hourly averages for all other variables. This report contains all data collected from 1 November 1992 through 31 October 1994. This provides the complete data record to date for the $\mathrm{Pu}^{\prime}$ - uanahulu and University of Hawai'i at Hilo (UHHilo) sites and continuity to previously reported data for the other stations. In this study, we analyze monthly total and average values summarized from the hourly measurements.

\section{RESULTS}

Climatological measurements during the $2 \mathrm{yr}$ study are summarized in Table 1. Missing periods of record for individual instruments (due to site vandalism or faulty sensors) are also given in Table 1. Evaporation was estimated using the Penman-Monteith method (Monteith 1965). While the data are in general agreement with previously published results, there are several noteworthy features of the climate cross-section. These are discussed below.

\section{Wind}

Seemingly indifferent to the synoptic trade wind field, surface flow fluctuates in a diurnal rhythm as the mountain 'breathes' each day. Both land-sea and land-free atmosphere temperature differences drive the daily thermal circulation that controls surface winds. Under light synoptic wind conditions, this daytime convergence

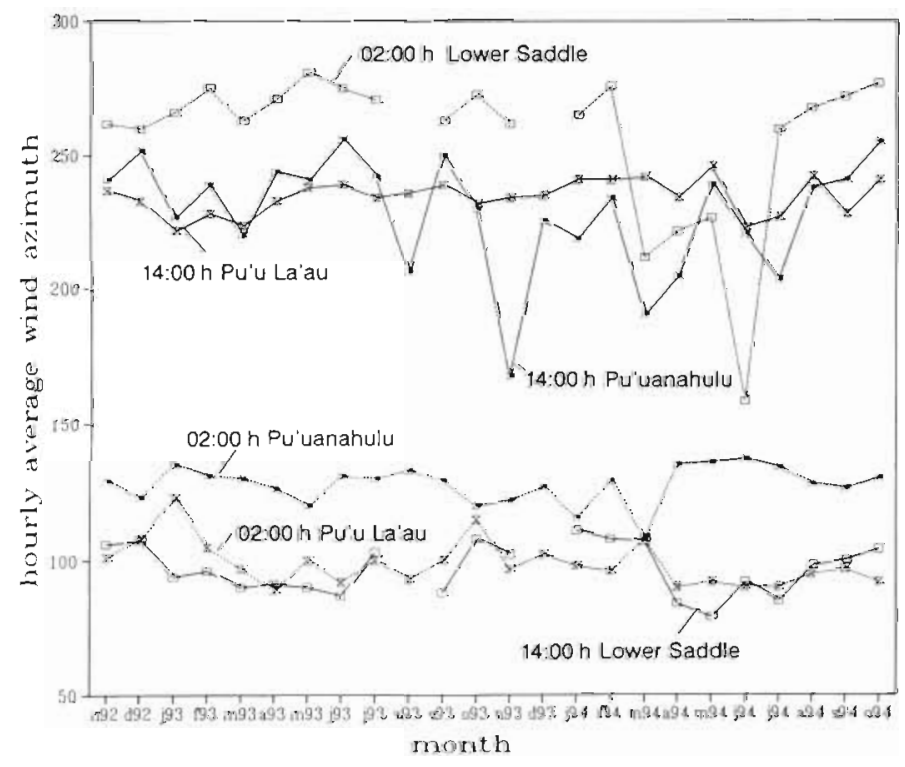

Fig. 2. Monthly average wind direction for the hours ending at 02:00 and 14:00 h HST (Hawalian Standard Time) showing the consistency of the diurnal wind reversal at all locations. Mirror image wind direction patterns occur of opposing windward (Lower Saddle) and leeward (Pu'uanahulu and Pu'u La'au) slopes 
of the winds toward the mountain summits can bring a torus of clouds and rain to the upper slopes. Fig. 2 shows the average wind direction for 02:00 and 14:00 h HST (Hawaiian Standard Time) for leeward and windward sites below the inversion and the leeward high elevation site. Daytime winds over our windward stations were almost due easterly, corresponding closely with results given by Chen \& Nash (1994) based on 40 d of measurements between 11 July and 24 August 1990. The correspondence is good for the nighttime winds at leeward sites as well, with winds directly downslope as would be expected under gravity drainage of cool air.

Our measurements differ, however, for the daytime winds at the leeward sites. As shown in Fig. 2, the daytime surface wind azimuth converges for the 2 leeward sites to approximately 220 to $230^{\circ}$. This trajectory is consistent with the heavy, upslope fog that predominates between the 2 locations on the leeward slopes of Mauna Kea. During Chen \& Nash's (1994) study, daytime winds near the Pu'uanahulu site averaged northwesterly (14:00 h HST), an upslope trajectory, rather than southwesterly, a parallel slope trajectory, as prevailed for all months except one during our study period.

Wind speed averaged less than $2 \mathrm{~m} \mathrm{~s}^{-1}$ for all sites except Pu'uanahulu (Table 1). At the leeward sites, maximum wind speeds at Pu'uanahulu (typically observed in early afternoon! nearly double those observed above the inversion at Pu'u La'au, despite virtually identical net radiation values (and by implication, identical surface air heating). This is presumably a consequence of a greater upslope area of heating for the lower site. The diurnal wind speed pattern is similar for all sites with daytime upslope wind speeds exceeding the nighttime drainage wind speed. As has been documented on Haleakalā, Maui (Giambelluca \& Nullet 1991), a dip in wind speed accompanies the diurnal wind direction reversals in early morning and late afternoon

\section{Moisture}

Diurnal wind patterns and the free atmosphere temperature and humidity profiles play major roles in the availability of water on the mountain slopes. The diurnal movement of air up and down slope carries with it a thin skin of source air. The cool, dry nighttime drainage winds [measured at $55 \mathrm{~m}$ depth by Mendonca (1969)] originate above the inversion while the daytime upslope winds bring a layer of moist, marine surface air (600 $\mathrm{m}$ depth; Mendonca 1969) through the inversion layer. This has the effect of creating opposing diurnal relative humidity patterns above and

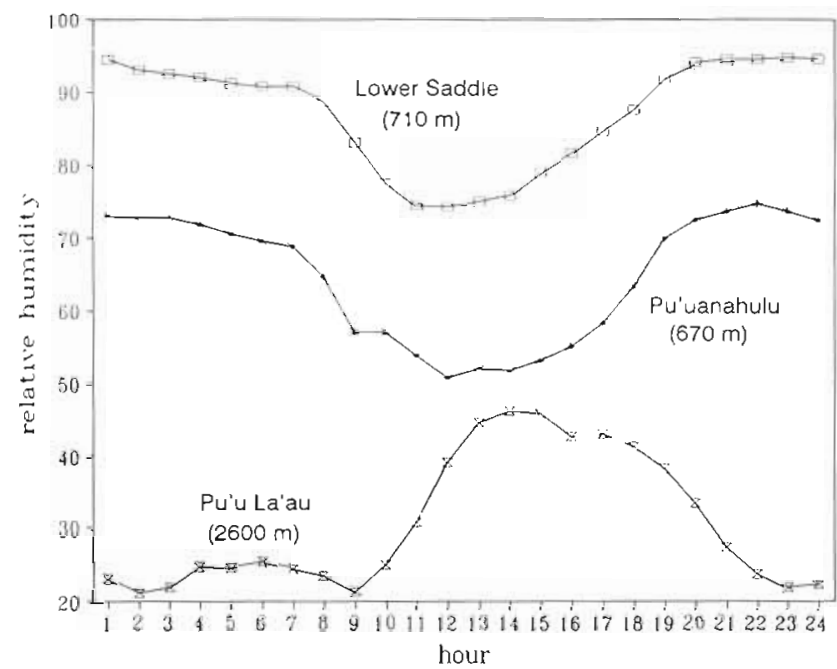

Fig. 3. Comparative diurnal relative humidity curves for February 1993. The humidity is lower on leeward slopes ( $\mathrm{Pu}^{\prime}-$ uanahulu) than windward slopes (Lower Saddle) at similar elevations. Above the inversion (Pu'u La'au), the diurnal pattern reverses, with higher relative humidity values during daylight hours as moist air penetrates the inversion layer and increases the vapor pressure at the site

below the inversion as shown in Fig. 3 for February 1993.

The diurnal thermal circulations account for most of the variance ( 54 to $90 \%$ ) in rainfall frequency over the island (Schroeder et al. 1977), producing afternoon maxima at most locations (associated with upslope winds and attendant cloud formation) and nocturnal maxima along windward coastlines as nighttime drainage winds converge with onshore trade wind flow. This pattern is interrupted during non-trade wind conditions and during periods when winter synoptic events, such as frontal passages and disturbances associated with upper-level troughs. In a comparison of rainfall totals at $\mathrm{Pu}$ 'u La'au and Pu'uanahulu to test the association of rainfall-producing mechanisms above and below the inversion, winter rainfall (October through March, largely a consequence of synoptic events) was highly correlated $(r=0.80)$, while summer rainfall (April through September, a consequence of local circulations under strong trade wind inversion conditions) showed virtually no correlation at all, i.e. rainfall occurred on different days with no significant correlation in amount.

Other sources of moisture to the surface include cloud water and dew. Direct interception of cloud droplets can provide a significant source of moisture above the cloud base at 600 to $800 \mathrm{~m}$. The ecological importance of cloud water interception increases as average rainfall decreases. At the Pu'u La'au site, for example, cloud water collection from a louvered fog 


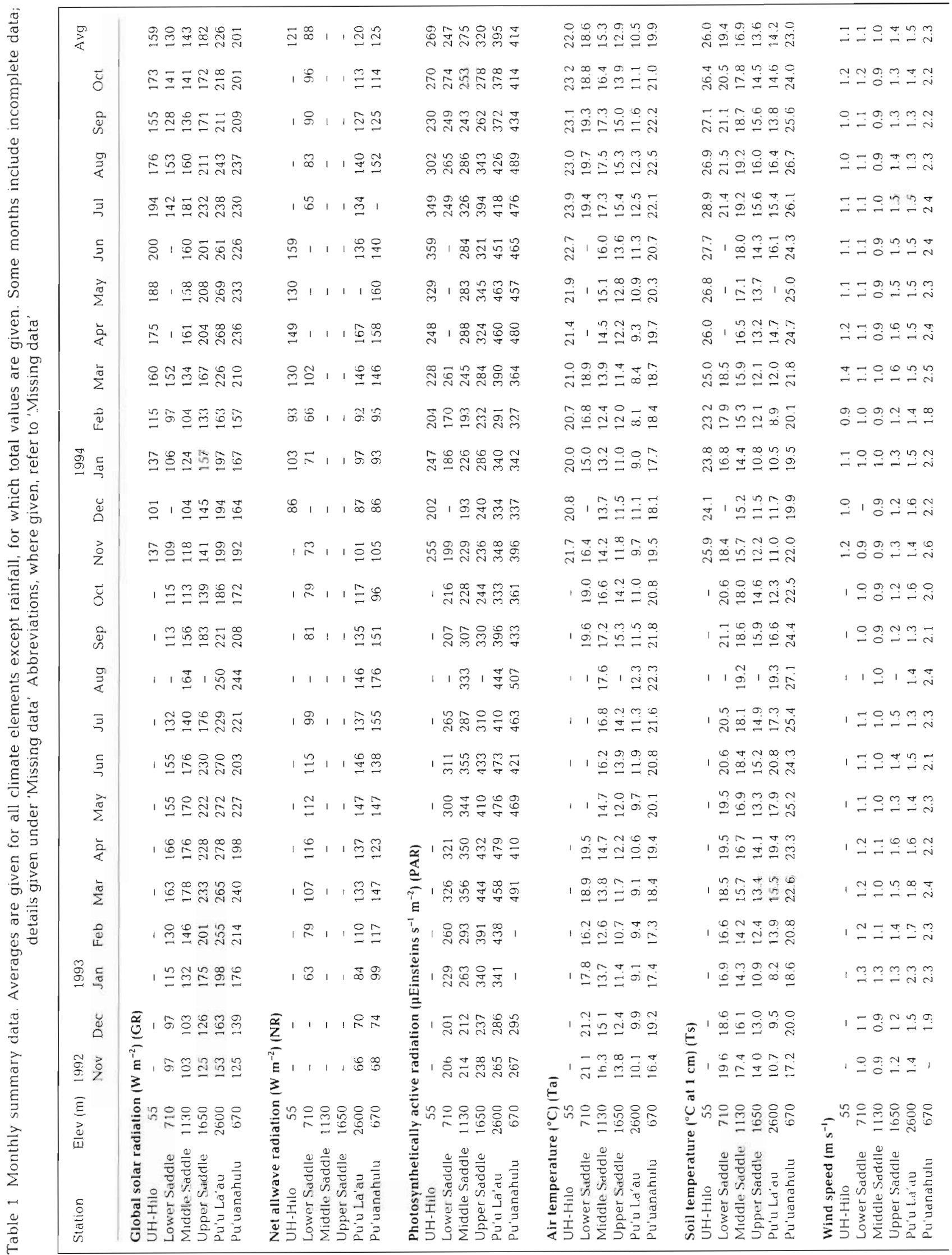




\begin{tabular}{|c|c|c|c|c|c|c|c|c|}
\hline 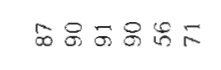 & 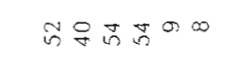 & 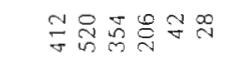 & 員䒺总 & \multicolumn{2}{|l|}{ 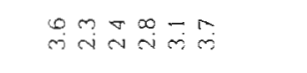 } & \multirow{11}{*}{\multicolumn{3}{|c|}{ 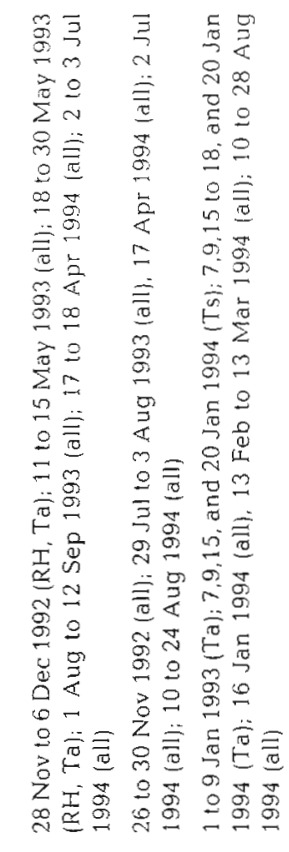 }} \\
\hline 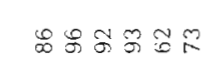 & 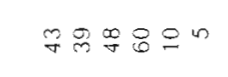 & 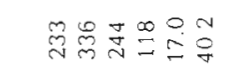 & 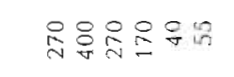 & \multicolumn{2}{|l|}{ 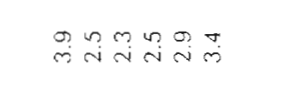 } & & & \\
\hline 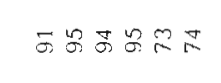 & $8985=$ & 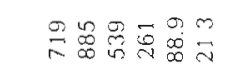 & 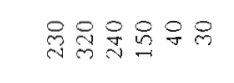 & \multicolumn{2}{|l|}{ 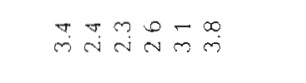 } & & & \\
\hline 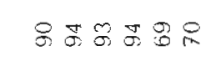 & 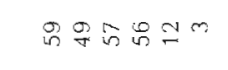 & 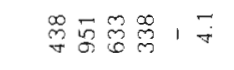 & 屏品品总的品 & \multicolumn{2}{|l|}{ 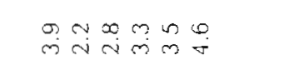 } & & & \\
\hline 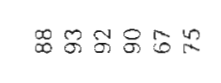 & 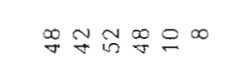 & 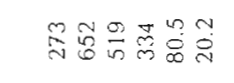 & 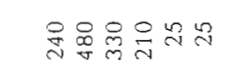 & \multicolumn{2}{|l|}{ 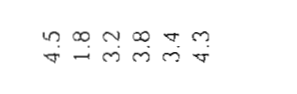 } & & & \\
\hline 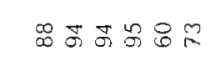 & $\stackrel{\infty}{i}, 8: 80$ in & 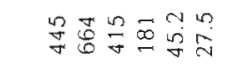 & 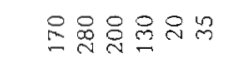 & \multicolumn{2}{|l|}{ 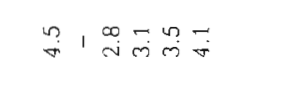 } & & & \\
\hline 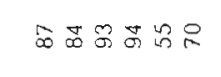 & 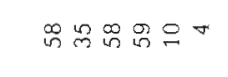 & 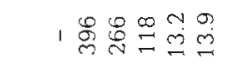 & 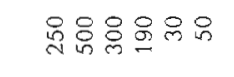 & \multicolumn{2}{|l|}{ 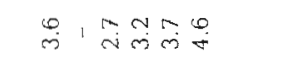 } & & & \\
\hline 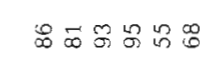 & 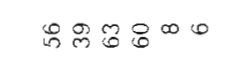 & 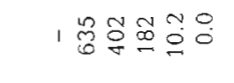 & 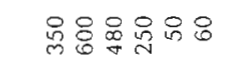 & \multicolumn{2}{|l|}{ 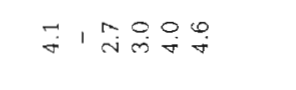 } & & & \\
\hline 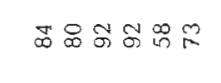 & 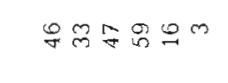 & 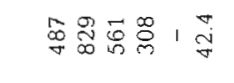 & 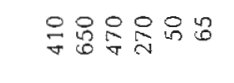 & \multicolumn{2}{|l|}{ 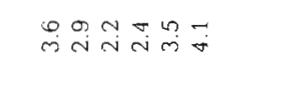 } & & & \\
\hline 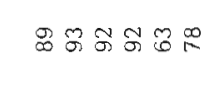 & 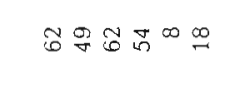 & 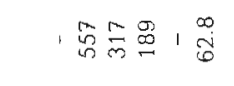 & 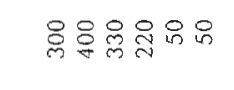 & \multicolumn{2}{|l|}{$\stackrel{b}{i}=\stackrel{0}{=}=\vec{i} \hat{i}$} & & & \\
\hline ஹహ్ & $\underset{\gamma}{\sim} \stackrel{\infty}{\leftrightarrow} \underset{\gamma}{*} \sigma 0$ & 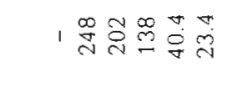 & 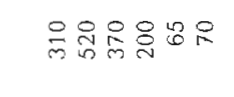 & \multicolumn{2}{|l|}{ 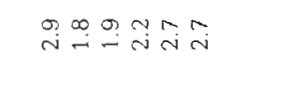 } & & & \\
\hline 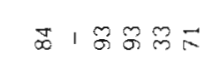 & 守 1 合触 $=$ & 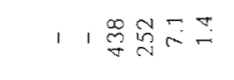 & 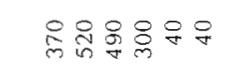 & \multicolumn{2}{|l|}{ 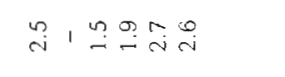 } & \multirow{4}{*}{ 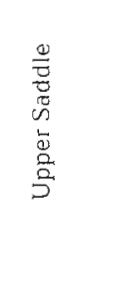 } & \multirow{4}{*}{ 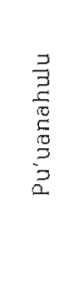 } & \multirow{4}{*}{ 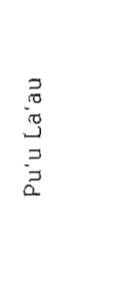 } \\
\hline 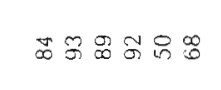 & 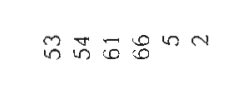 & 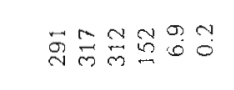 & 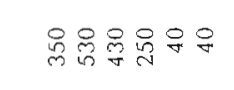 & \multicolumn{2}{|l|}{$\bar{m}-\stackrel{\infty}{-} \sigma \vec{\sim}$} & & & \\
\hline 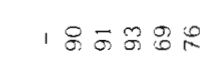 & 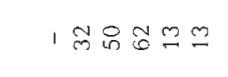 & , & 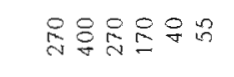 & \multicolumn{2}{|l|}{ | } & & & \\
\hline 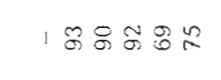 & $19 \overline{7}=0$ & 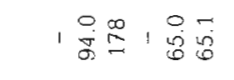 & 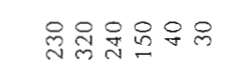 & \multicolumn{2}{|l|}{ | } & & & \\
\hline 1 ब1 용 & 118,00 & , 1 兽 1 & 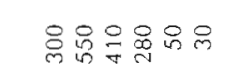 & \multicolumn{2}{|l|}{ 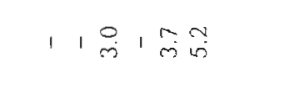 } & \multirow{4}{*}{\multicolumn{3}{|c|}{ 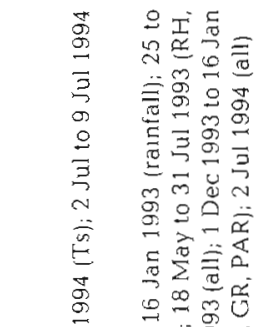 }} \\
\hline 1 ㅅํㅇㅛㅠ & 19ำ요웅 & 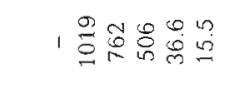 & 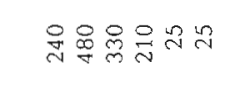 & \multicolumn{2}{|l|}{ 1 } & & & \\
\hline 1 1 & 1 专台怘 $=\infty$ & 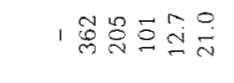 & 욤웜윰욕요 & \multicolumn{2}{|l|}{ 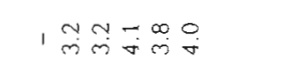 } & & & \\
\hline | எல் & 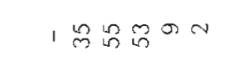 & 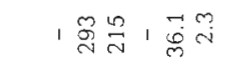 & 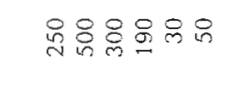 & \multicolumn{2}{|l|}{ 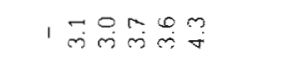 } & & & \\
\hline 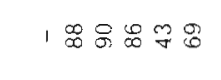 & 1 幽品昌以。 & | & 品品品品的 & \multicolumn{2}{|l|}{ 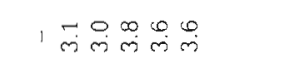 } & \multirow{6}{*}{ 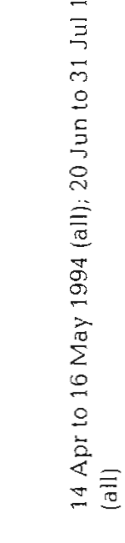 } & & \\
\hline 1 & 1 망유 & | & 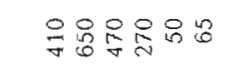 & \multicolumn{2}{|l|}{ 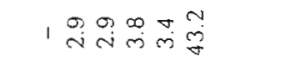 } & & & \\
\hline 1098 & 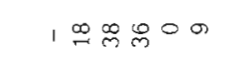 & , $\begin{array}{l}9 \\
0\end{array}$ & 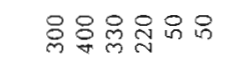 & \multicolumn{2}{|l|}{ 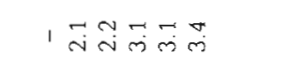 } & & & \\
\hline 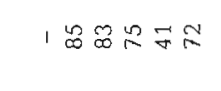 & 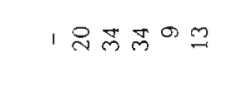 & 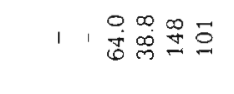 & 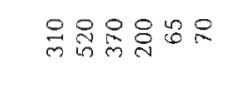 & \multicolumn{2}{|l|}{ 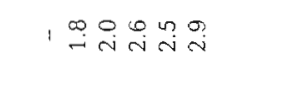 } & & & \\
\hline オே品品司 & 1 舟的的里 & 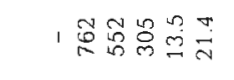 & 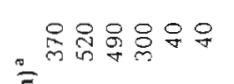 & \multirow{3}{*}{ 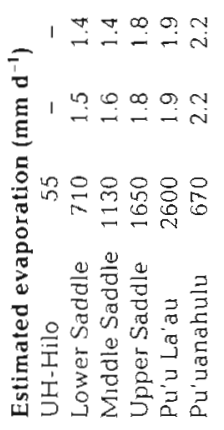 } & \multirow{3}{*}{ 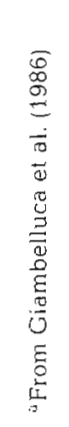 } & & & $\sum_{0}$ \\
\hline 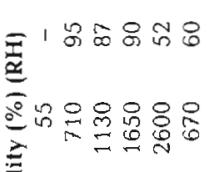 & 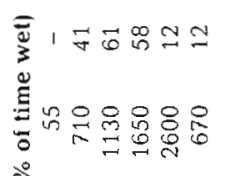 & 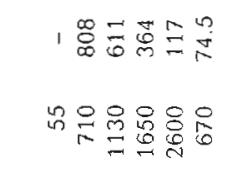 & 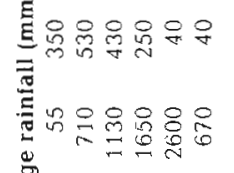 & & & & & $\therefore \delta$ \\
\hline 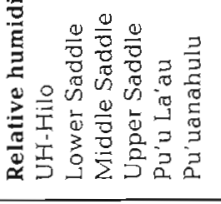 & 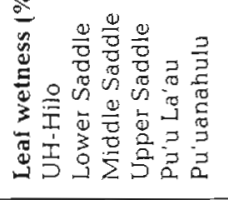 & 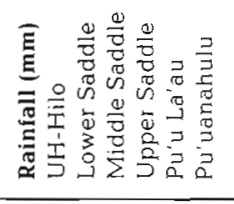 & 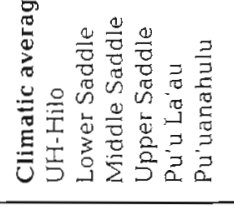 & & & 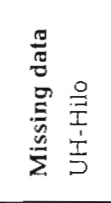 & 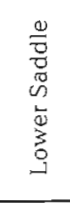 & 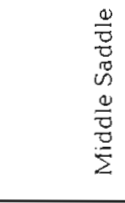 \\
\hline
\end{tabular}




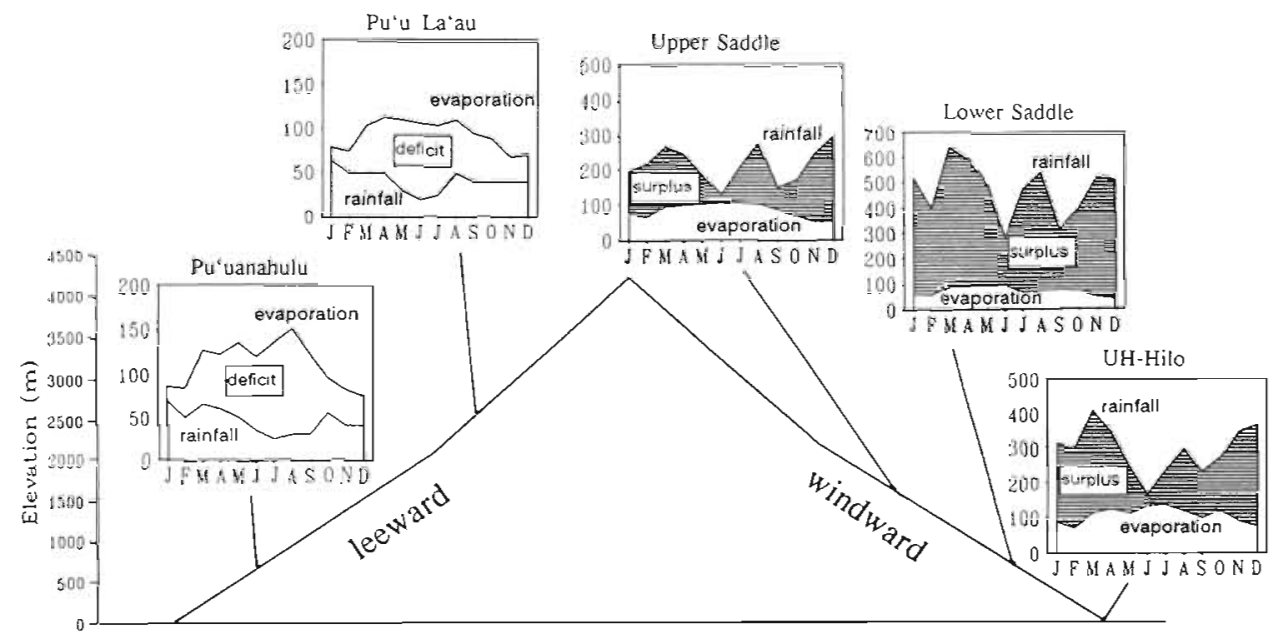

Fig. 4. Climate diagrams for the mountain cross-section. On average, rainfall greatly exceeds evaporation on windward slopes, while the reverse is true of leeward areas

gauge $(25.8 \mathrm{~mm})$ exceeded rainfall $(17.5 \mathrm{~mm})$ during an exceptionally dry 5 mo period, February through June 1992 (Juvik \& Nullet 1995). Dew does not appear to be an ecologically significant source of moisture at the dry leeward sites in this study, as suggested by negligible nighttime leaf wetness frequencies. High nighttime relative humidity values suggest dew formation at the wet windward sites, although at these high rainfall locations, vegetation seldom experiences moisture deficits and thus would not necessarily benefit by added moisture from dew and cloud water.

The atmospheric evaporative demand determines, in part, moisture availability and stress on vegetation. On windward slopes, rainfall exceeded estimated evaporative demand for all months except January and February 1993, and March 1993 at $1650 \mathrm{~m}$. For leeward locations, estimated evaporative demand exceeded rainfall during 21 of the 24 months at the $2600 \mathrm{~m}$ elevation site, and 22 of 24 months at the $670 \mathrm{~m}$ elevation site. Consistent with implied high moisture stress, both areas support dryland vegetation: open forest with bunch grass ground cover. The relationship between rainfall and estimated evaporation under normal climatic conditions is illustrated in Fig. 4. This figure compares only the magnitudes of rainfall and evaporation and generally sets an upper limit on the average moisture availability to plants as a complete water balance would show that much of the rainfall input quickly infiltrates into the porous substrate and is not available to meet the evaporative demand.

\section{Radiation}

Fig. 5 shows elevation profiles of measured solar radiation as a percentage of modeled clear day values
(Bird \& Riordan 1986). On windward slopes, maximum attenuation of solar radiation occurs at $700 \mathrm{~m}$, near the rainfall maximum (and, by inference, the cloud cover maximum). Results correspond well with similar measurements reported for Maui, although attenuation at $2600 \mathrm{~m}$ exceeds that for a comparable exposure and elevation on Haleakalā (Giambelluca \& Nullet 1991), suggesting greater cloudiness on the larger mountain. The contrast between the attenuation of solar radiation on upper and lower mountain slopes is much greater on windward than leeward exposures.

At $\mathrm{Pu}^{\prime} u$ anahulu, net radiation exceeded the average value at $\mathrm{Pu}$ 'u La'au despite receiving about $11 \%$ less

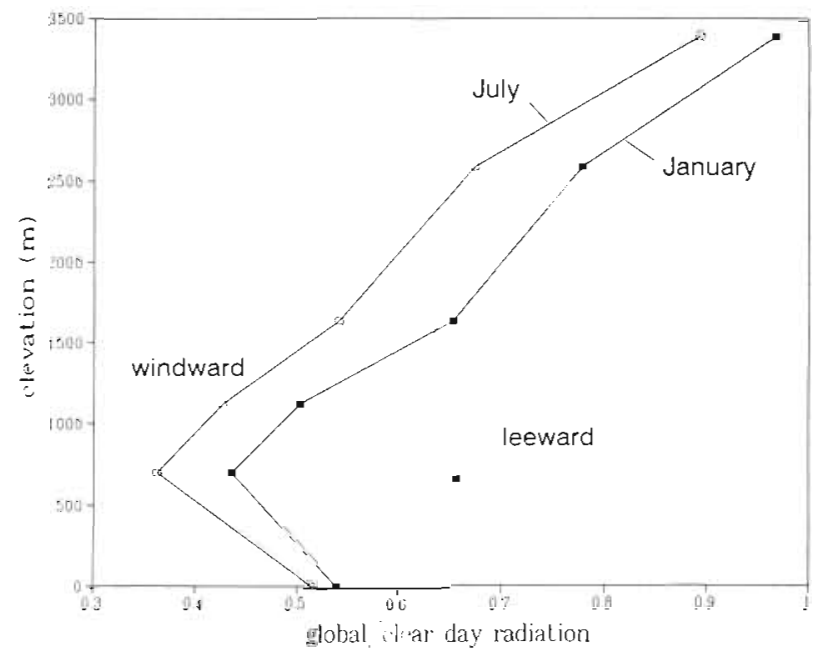

Fig. 5. Measured solar radiation as a percentage of clear-day radiation for January and July showing the reduction by cloud cover. Data for $3400 \mathrm{~m}$ are long-term averages for Mauna Loa Observatory (see Fig. 1) for comparison. Annually, clear skies occur more frequently during the cooler winter months, while in summer convective heating and stronger trade winds lead to increased cloud cover 
solar radiation, a consequence of generally clearer skies and less far infrared absorption in the dry air above the inversion. For example, nighttime longwave radiation loss at Pu'u La'au averaged about $20 \mathrm{~W} \mathrm{~m}^{-2}$ higher than at $\mathrm{Pu}$ uanahulu. At comparable elevations, net radiation averaged a higher fraction of solar radiation at the windward Lower Saddle site than at the leeward Pu'uanahulu site. The photosynthetically active component generally decreased as a percentage of solar radiation with elevation within the range of 35 to $45 \%$.

\section{Temperature}

The annual range in average monthly air temperature was similar (less than $6^{\circ} \mathrm{C}$ ) at all sites, although the soil temperature range stratifies by elevation and exposure. At the wet windward sites, average air and soil temperatures are nearly equal, indicating that the air layer near the ground is in thermal equilibrium with the surface. On leeward slopes, however, soil temperature greatly exceeds air temperature as a consequence of lower soil moisture and less canopy shading of the surface. For the 2 leeward sites, despite receiving lower net radiation and higher rainfall during the study period, the soil-air temperature difference was greater at the Pu'u La'au (2600 m) than at Pu'uanahulu $(670 \mathrm{~m})$. Presumably, the flow of cool, free atmosphere air greatly retards air temperatures near the surface at Pu'u La'au.

\section{CONCLUSION}

This study has presented the results of 2 yr of surface meteorological observations transecting Hawai'i Island including stations on wet windward slopes, and dry, leeward sites above and below the trade wind inversion. The data illustrate the striking climate gradients found in Hawailan mountains. The data has expanded on previously reported results for the wet windward sites and the high elevation Pu'u La'au site. These are the first complete climatic average data reported for the dry leeward slopes of Mauna Loa.

Editor: V. Meentemeyer, Athens, Georgia, USA
Acknowledgements. Financial support was provided by the National Science Foundation through Grant DEB 8918526 (Ecosystem Dynamics in Hawai'i), principal investigator D. Mueller-Dombois, and by the U.S. Fish and Wildlife Service.

\section{LITERATURE CITED}

Armstrong RW (ed) (1983) A.tlas of Hawaii, 2nd edn. University of Hawai'i Press, Honolulu

Bird RE, Riordan C (1986) Simple solar spectral model for direct and diffuse irradiance on horizontal and tilted planes at the earth's surface for cloudless atmospheres. J Climate appl Meteorol 25:87-97

Blumenstock DI, Price S (1967) Climates of the states: Hawaii Climatography of the United States No 60-51 US Dept Commerce, Washington, DC

Chen YL, Nash AJ (1994) Diurnal variation of surface airflow and rainfall frequencies on the Island of Hawaii. Mon Weather Rev 122:34-56

Chen YL, Wang JJ (1994) Diurnal variation of surface thermodynamic fields on the island of Hawaii. Mon Weather Rev 122:2125-2138

Ekern PC (1983) Measured evaporation in high rainfall areas, leeward Koolau Range, Oahu, Hawaii. Water Resours Res Center Tech Rep 156, Universtiy of Hawaii, Honolulu

Giambelluca TW, Nullet D (1991) Influence of the trade wind inversion on the climate of a leeward mountain slope in Hawaii. Clim Res 1:207-216

Glambelluca, TW, Nullet, MA, Schroeder, TA (1986) Rainfall Atlas of Hawaii. Depart Land Nat Resour Rep R76, State of Hawaii, Honolulu

Juvik JO, Nullet D (1994) A climate transect through tropical montane rainforest in Hawaii. $J$ appl Meteorol 33:1304-1312.

Juvik JO, Nullet D (1995) Relationships between rainfall, cloud-water interception, and canopy throughfall in a Hawaiian montane forest. In: Hamilton LS, Juvik JO, Scatena FN (eds) Tropical montane cloud forests. Springer-Verlag, New York, p 165-182

Juvik JO, Nullet D, Banko P, Hughes K (1993) Forest climatology near the tree line in Hawai'i. Agric For Meteorol 66: $159-172$

Mendonca BG (1969) Local wind circulation on the slopes of Mauna Loa. J appl Meteorol 8:533-541

Monteith JL (1965) Evaporation and environment. Proc Symp Soc exp Biol 19:205-234

Nullet D (1989) Influence of a tropical island mountain on solar radiation, air temperature, and vapor pressure. $J$ appl Meteorol 28:233-238

Schroeder TA (1993) Climate controls. In: Sanderson M (ed) Prevailing trade winds. University of Hawaii Press, Honolulu

Schroeder TA, Kilonsky BJ, Meisner BN (1977) Diurnal variation in rainfall and cloudiness. UHMET Rep 77-03, Department of Meteorology, University of Hawaii, Honolulu

Manuscript first received: January 22, 1995

Revised version accepted: March 3, 1995 